Pacific

Journal of

Mathematics

\title{
GRADIENT ESTIMATES
}

FOR A NONLINEAR LICHNEROWICZ EQUATION

UNDER GENERAL GEOMETRIC FLOW ON COMPLETE NONCOMPACT MANIFOLDS

LiANG ZHAO AND SHOUWEN FANG 


\title{
GRADIENT ESTIMATES \\ FOR A NONLINEAR LICHNEROWICZ EQUATION UNDER GENERAL GEOMETRIC FLOW ON COMPLETE NONCOMPACT MANIFOLDS
}

\author{
LiANG ZHAO AND SHOUWEN FANG
}

We study gradient estimates for positive solutions to the nonlinear parabolic equation

$$
\frac{\partial u}{\partial t}=\Delta u+c u^{-\alpha}
$$

under general geometric flow on complete noncompact manifolds, where $\alpha, c$ are two real constants and $\alpha>0$. As an application, we give the corresponding Harnack inequality.

\section{Introduction}

Recently, there has been active interest in the study of gradient estimates for partial differential equations on noncompact manifolds. Wu [2010] gave a local $\mathrm{Li}-\mathrm{Yau}$ type gradient estimate for positive solutions to a general nonlinear parabolic equation

$$
u_{t}=\Delta u-\nabla \varphi \nabla u-a u \log u-q u
$$

in $M \times[0, \tau]$, where $a \in R, \varphi$ is a $C^{2}$-smooth function and $q=q(x, t)$ is a function, which generalizes many previous well-known gradient estimates. Zhu [2011] investigated the fast diffusion equation

$$
u_{t}=\Delta u^{\alpha} \quad(0<\alpha<1) .
$$

Theorem 1.1 [Zhu 2011]. Let $M$ be a Riemannian manifold of dimension $n \geq 2$ with $\operatorname{Ric} M \geq-k$ for some $k \geq 0$. Suppose that $v=-(\alpha /(\alpha-1)) u^{\alpha-1}$ is any positive solution to (1-1) in $Q_{R, T} \equiv B\left(x_{0}, R\right) \times\left[t_{0}-T, t_{0}\right] \subset M \times(-\infty, \infty)$. Suppose also that $v \leq \tilde{M}$ in $Q_{R, T}$. Then there exists a constant $C=C(\alpha, M)$ such that

$$
\frac{|\nabla v|}{v^{1 / 2}} \leq C \tilde{M}^{1 / 2}\left(\frac{1}{R}+\frac{1}{\sqrt{T}}+\sqrt{k}\right)
$$

in $Q_{\frac{R}{2}, \frac{T}{2}}$.

MSC2010: primary 58J05; secondary 58J35.

Keywords: gradient estimates, geometric flow, Harnack inequality. 
Later, Huang and Li [2014] studied the generalized equation

$$
u_{t}=\Delta_{f} u^{\alpha} \quad(\alpha>0)
$$

on Riemannian manifolds and got some interesting gradient estimates, where $f$ is a smooth function and $\Delta_{f}$ is defined by

$$
\Delta_{f}=\Delta-\nabla f \cdot \nabla .
$$

For the elliptic case, Zhang and Ma [2011] considered the equation

$$
\Delta_{f} u+c u^{-\alpha}=0 \quad(\alpha>0)
$$

on complete noncompact manifolds when the constant $N$ is finite and the $N$ Bakry-Émery Ricci tensor is bounded from below, obtaining the following gradient estimate.

Theorem 1.2 [Zhang and Ma 2011]. Suppose (M,g) is a complete noncompact $n$-dimensional Riemannian manifold with $N$-Bakry-Émery Ricci tensor bounded from below by the constant $-K=:-K(2 R)$, where $R>0$ and $K(2 R)>0$ in the metric ball $B_{2 R}(p)$ around $p \in M$. Let $u$ be a positive solution of (1-2). Then

(1) if $c>0$, we have

$$
\begin{array}{r}
\frac{|\nabla u|^{2}}{u^{2}}+c u^{-(\alpha+1)} \leq \frac{(N+n)(N+n+2) c_{1}^{2}}{R^{2}}+\frac{(N+n)\left((N+n-1) c_{1}+c_{2}\right)}{R^{2}} \\
+\frac{(N+n) \sqrt{(N+n) K} c_{1}}{R}+2(N+n) K,
\end{array}
$$

(2) if $c<0$, we have

$$
\begin{aligned}
\frac{|\nabla u|^{2}}{u^{2}}+c u^{-(\alpha+1)} \leq(A+\sqrt{A})|c|\left(\inf _{B_{p}(2 R)} u\right)^{-\alpha-1}+\frac{(N+n)\left((N+n-1) c_{1}+c_{2}\right)}{R^{2}} & \\
+ & \frac{(N+n) c_{1}^{2}}{R^{2}}\left(n+N+2+\frac{n+N}{2 \sqrt{A}}\right) \\
& +\frac{(N+n) \sqrt{(N+n) K} c_{1}}{R}+\left(2+\frac{1}{\sqrt{A}}\right)(n+N) K,
\end{aligned}
$$

where $A=(N+n)(\alpha+1)(\alpha+2)$ and $c_{1}, c_{2}$ are absolute positive constants.

For interesting gradient estimates on manifolds with fixed metric, see [Chen and Chen 2009; 2010; Li 2005; Ma 2006; 2010; Zhao 2013; 2014].

However, in the above works, the authors considered gradient estimates for positive solutions to nonlinear equations on complete noncompact manifolds with fixed metric, so it is natural to ask how gradient estimates vary if the metric on a manifold evolves with time. In Perelman's breakthrough work [2002] on the 
Poincare conjecture, the author showed the gradient estimate for the fundamental solution of the conjugate heat equation

$$
\Delta u-R u+\partial_{t} u=0
$$

under Ricci flow on a closed Riemannian manifold $M$, where $R$ is the scalar curvature. Since then, a large amount work has been done to study gradient estimates along geometric flow for the solution of the nonlinear equation. Kuang and Zhang [2008] established the corresponding pointwise gradient estimate. For the heat equation under Ricci flow, Liu [2009] got first-order gradient estimates for its positive solutions and derived Harnack inequalities and second-order gradient estimates. Later, Sun [2011] extended it to general geometric flow.

Since gradient estimates often lead to Liouville type theorems and Harnack inequalities, which played an important role in the proof of the Poincaré conjecture, for nonlinear heat equations on manifolds, to get good control of suitable Harnack quantities (depending on nonlinear terms), one may need the key lower bound assumption about Ricci curvature. The results of Theorem 1.2 are about gradient estimates for the elliptic equation (1-2). In this paper, we will extend these results to the parabolic variant of the problem. Thus, we consider the equation

$$
\frac{\partial u}{\partial t}=\Delta u+c u^{-\alpha}
$$

on complete noncompact manifolds $M$ with evolving metric, where $\alpha, c$ are two real constants and $\alpha>0$. The motivation for this paper is that (1-3) can be viewed as a simple parabolic Lichnerowicz equation. It is well known that the Lichnerowicz equation arises from the Hamiltonian constraint equation for the Einstein-scalar field. Since (1-3) contains a negative power nonlinearity, it is interesting to discuss gradient estimates for it.

We state our main results about (1-3) as follows.

Theorem 1.3. Let $(M, g(t))$ be a smooth one-parameter family of complete Riemannian manifolds evolving by

$$
\frac{\partial}{\partial t} g=2 h
$$

for $t$ in some time interval $[0, T]$. Let $M$ be complete under the initial metric $g(0)$. Given $x_{0} \in M$ and $R>0$, let $u$ be a positive solution to the nonlinear equation

$$
\frac{\partial u}{\partial t}=\Delta u+c u^{-\alpha}
$$

in the cube $Q_{2 R, T}:=\left\{(x, t) \mid d\left(x, x_{0}, t\right) \leq 2 R, 0 \leq t \leq T\right\}$. Suppose that there exist constants $K_{1}, K_{2}, K_{3}, K_{4} \geq 0$ such that

$$
\text { Ric } \geq-K_{1} g, \quad-K_{2} g \leq h \leq K_{3} g, \quad|\nabla h| \leq K_{4}
$$


on $Q_{2 R, T}$. Then for $(x, t) \in Q_{R, T}$ and positive constants $c_{1}, c_{2}$,

(1) if $c<0$ and for a positive constant $\tilde{M}, u^{-(\alpha+1)} \leq \tilde{M}$ for all $(x, t) \in M \times[0, T]$, we have

$$
\beta \frac{|\nabla u|^{2}}{u^{2}}+c u^{-(\alpha+1)}-\frac{u_{t}}{u} \leq H_{1}+H_{2}+\frac{n}{\beta} \frac{1}{t}
$$

where

$$
\begin{aligned}
& H_{1}= \frac{n}{\beta}\left(\frac{(n-1)\left(1+\sqrt{K_{1}} R\right) c_{1}^{2}+c_{2}+2 c_{1}^{2}}{R^{2}}+\sqrt{c_{3}} K_{2}-c(\alpha+1) \tilde{M}+\frac{n c_{1}^{2}}{2 \beta(1-\beta) R^{2}}\right), \\
& H_{2}=\left(\frac{n^{2}}{4 \beta^{2}(1-\beta)^{2}}\left(2 \beta K_{1}+2(1-\beta) K_{3}-c(\beta+\alpha)(\alpha+1) \tilde{M}+\frac{3}{2} K_{4}\right)^{2}\right. \\
&\left.+\frac{n^{2}}{\beta}\left(\left(K_{2}+K_{3}\right)^{2}+\frac{3}{2} K_{4}\right)\right)^{\frac{1}{2}},
\end{aligned}
$$

(2) if $c>0$, we have

$$
\beta \frac{|\nabla u|^{2}}{u^{2}}+c u^{-(\alpha+1)}-\frac{u_{t}}{u} \leq \tilde{H}_{1}+\tilde{H}_{2}+\frac{n}{\beta} \frac{1}{t},
$$

where

$$
\begin{aligned}
& \tilde{H}_{1}=\frac{n}{\beta}\left(\frac{(n-1)\left(1+\sqrt{K_{1}} R\right) c_{1}^{2}+c_{2}+2 c_{1}^{2}}{R^{2}}+\sqrt{c_{3}} K_{2}+\frac{n c^{2}}{2 \beta(1-\beta) R^{2}}\right), \\
& \tilde{H}_{2}=\left(\frac{n^{2}}{4 \beta^{2}(1-\beta)^{2}}\left(2 \beta K_{1}+2(1-\beta) K_{3}+\frac{3}{2} K_{4}\right)^{2}+\frac{n^{2}}{\beta}\left(\left(K_{2}+K_{3}\right)^{2}+\frac{3}{2} K_{4}\right)\right)^{\frac{1}{2}} .
\end{aligned}
$$

Here $0<\beta<1, c_{1}, c_{2}, c_{3}$ are positive constants.

Remark. In fact, our result is the parabolic version of Theorem 1.2 under the evolving metric.

Letting $R \rightarrow \infty$, we can get the following global gradient estimate for the nonlinear parabolic equation (1-3).

Corollary 1.4. Let $(M, g(0))$ be a complete noncompact Riemannian manifold without boundary, and suppose $g(t)$ evolves by (1-4) for $t \in[0, T]$ and satisfies

$$
\text { Ric } \geq-K_{1} g, \quad-K_{2} g \leq h \leq K_{3} g, \quad|\nabla h| \leq K_{4} .
$$

If $u$ is a positive solution to (1-3), then for $(x, t) \in M \times[0, T]$,

(1) if $c<0$ and $u^{-(\alpha+1)} \leq \tilde{M}$ for all $(x, t) \in M \times[0, T]$, we have

$$
\beta \frac{|\nabla u|^{2}}{u^{2}}+c u^{-(\alpha+1)}-\frac{u_{t}}{u} \leq \bar{H}_{1}+H_{2}+\frac{n}{\beta} \frac{1}{t},
$$

where

$$
\bar{H}_{1}=\sqrt{c_{3}} K_{2}-\frac{n}{\beta} c(\alpha+1) \tilde{M},
$$


(2) if $c>0$, we have

$$
\beta \frac{|\nabla u|^{2}}{u^{2}}+c u^{-(\alpha+1)}-\frac{u_{t}}{u} \leq \hat{H}_{1}+\tilde{H}_{2}+\frac{n}{\beta} \frac{1}{t}
$$

where $\hat{H}_{1}=\sqrt{c_{3}} K_{2}$ and $H_{2}, \tilde{H}_{2}$ are the same as in Theorem 1.3.

As an application, we get the following Harnack inequality.

Theorem 1.5. Let $(M, g(0))$ be a complete noncompact Riemannian manifold without boundary, and suppose $g(t)$ evolves by (1-4) for $t \in[0, T]$ and satisfies

$$
\text { Ric } \geq-K_{1} g, \quad-K_{2} g \leq h \leq K_{3} g, \quad|\nabla h| \leq K_{4} .
$$

Let $u$ be a positive solution to (1-3) with $u^{-(\alpha+1)} \leq \tilde{M}$ for all $(x, t) \in M \times(0, T]$. Then for any points $\left(x_{1}, t_{1}\right)$ and $\left(x_{2}, t_{2}\right)$ on $M \times(0, T]$ with $0<t_{1}<t_{2}$, we have the following Harnack inequality:

(1) if $c<0$, we have

$$
u\left(x_{1}, t_{1}\right) \leq u\left(x_{2}, t_{2}\right)\left(\frac{t_{2}}{t_{1}}\right)^{n / \beta} e^{\varphi\left(x_{1}, x_{2}, t_{1}, t_{2}\right)+\left(\bar{H}_{1}+H_{2}\right)\left(t_{2}-t_{1}\right)},
$$

(2) if $c>0$, we have

$$
u\left(x_{1}, t_{1}\right) \leq u\left(x_{2}, t_{2}\right)\left(\frac{t_{2}}{t_{1}}\right)^{n / \beta} e^{\varphi\left(x_{1}, x_{2}, t_{1}, t_{2}\right)+\left(c \tilde{M}+\hat{H}_{1}+\tilde{H}_{2}\right)\left(t_{2}-t_{1}\right)},
$$

where $\varphi\left(x_{1}, x_{2}, t_{1}, t_{2}\right)=\inf _{\gamma} \int_{t_{1}}^{t_{2}} \frac{1}{4 \beta}|\dot{\gamma}|^{2} d t$ and $\gamma$ is any spacetime path joining $\left(x_{1}, t_{2}\right)$ and $\left(x_{2}, t_{2}\right)$.

\section{Proof of Theorem 1.3}

Let $u$ be a positive solution to (1-3). Set $w=\log u$; then $w$ satisfies

$$
w_{t}=\Delta w+|\nabla w|^{2}+c e^{-w(\alpha+1)} .
$$

Lemma 2.1 [Sun 2011]. Suppose the metric evolves by (1-4). Then, for any smooth function $w$, we have

$$
\frac{\partial}{\partial t}|\nabla w|^{2}=-2 h(\nabla w, \nabla w)+2 \nabla w \nabla w_{t}
$$

and

$$
\frac{\partial}{\partial t} \Delta w=\Delta \frac{\partial}{\partial t} w-2 h \nabla^{2} w-2 \nabla w\left(\operatorname{div} h-\frac{1}{2} \nabla\left(\operatorname{tr}_{g} h\right)\right),
$$

where div $h$ is the divergence of $h$. 
Lemma 2.2. Assume $(M, g(t))$ satisfies the hypotheses of Theorem 1.3. We have

$$
\begin{aligned}
\left(\Delta-\frac{\partial}{\partial t}\right) F \geq- & 2 \nabla w \nabla F+t\left(\frac{\beta}{n}\left(|\nabla w|^{2}+c(\alpha+1) e^{-w(\alpha+1)}-w_{t}\right)^{2}\right. \\
+\left((\beta+\alpha) c(\alpha+1) e^{-w(\alpha+1)}+2(\beta-1) K_{3}-2 \beta K_{1}-\frac{3}{2} K_{4}\right)|\nabla w|^{2} & \left.-n\left(\frac{1}{\beta}\left(K_{2}+K_{3}\right)^{2}+\frac{3}{2} K_{4}\right)\right) \\
+ & c(\alpha+1) e^{-w(\alpha+1)} F-\frac{F}{t},
\end{aligned}
$$

where $F=t\left(\beta|\nabla w|^{2}+c e^{-w(\alpha+1)}-w_{t}\right)$ and $0<\beta<1$.

Proof. Define $F=t\left(\beta|\nabla w|^{2}+c e^{-w(\alpha+1)}-w_{t}\right)$. It is well known that for the Ricci tensor, we have the Bochner formula:

$$
\Delta|\nabla w|^{2} \geq 2\left|\nabla^{2} w\right|^{2}+2 \nabla w \nabla(\Delta w)-2 K_{1}|\nabla w|^{2} .
$$

Noting that

$$
\begin{aligned}
\Delta w_{t}= & (\Delta w)_{t}+2 h \nabla^{2} w+2 \nabla w\left(\operatorname{div} h-\frac{1}{2} \nabla\left(\operatorname{tr}_{g} h\right)\right) \\
= & -\left(|\nabla w|^{2}\right)_{t}+c(\alpha+1) e^{-w(\alpha+1)} w_{t}+w_{t}+2 h \nabla^{2} w+2 \nabla w\left(\operatorname{div} h-\frac{1}{2} \nabla\left(\operatorname{tr}_{g} h\right)\right) \\
= & 2 h(\nabla w, \nabla w)-2 \nabla w \nabla w_{t}+c(\alpha+1) e^{-w(\alpha+1)} w_{t} \\
& \quad+w_{t t}+2 h \nabla^{2} w+2 \nabla w\left(\operatorname{div} h-\frac{1}{2} \nabla\left(\operatorname{tr}_{g} h\right)\right), \\
\Delta w= & -|\nabla w|^{2}-c e^{-w(\alpha+1)}+w_{t}=\left(1-\frac{1}{\beta}\right)\left(-c e^{-w(\alpha+1)}+w_{t}\right)-\frac{F}{\beta t},
\end{aligned}
$$

we have

$$
\begin{gathered}
\Delta F=t\left(\beta \Delta|\nabla w|^{2}+c \Delta e^{-w(\alpha+1)}-\Delta w_{t}\right) \\
=t\left(\beta \Delta|\nabla w|^{2}\right)+t c\left((\alpha+1)^{2} e^{-w(\alpha+1)}|\nabla w|^{2}-(\alpha+1) e^{-w(\alpha+1)} \Delta w\right)-t \Delta w_{t} \\
\geq t\left(2 \beta\left|\nabla^{2} w\right|^{2}+2 \beta \nabla w \nabla(\Delta w)-2 K_{1} \beta|\nabla w|^{2}+c(\alpha+1)^{2} e^{-w(\alpha+1)}|\nabla w|^{2}\right. \\
-c(\alpha+1) e^{-w(\alpha+1)}\left(\left(1-\frac{1}{\beta}\right)\left(-c e^{-w(\alpha+1)}+w_{t}\right)-\frac{F}{\beta t}\right)+\left(|\nabla w|^{2}\right)_{t} \\
\left.\quad-c(\alpha+1) e^{-w(\alpha+1)} w_{t}-w_{t t}-2 h \nabla^{2} w-2 \nabla w\left(\operatorname{div} h-\frac{1}{2} \nabla\left(\operatorname{tr}_{g} h\right)\right)\right) \\
=t\left(2 \beta\left|\nabla^{2} w\right|^{2}-\frac{2}{t} \nabla w \nabla F+2 \beta \nabla w \nabla w_{t}-2 h(\nabla w, \nabla w)\right. \\
\quad+\left((2 \beta+\alpha-1) c(\alpha+1) e^{-w(\alpha+1)}-2 K_{1} \beta\right)|\nabla w|^{2} \\
\quad+c^{2}(\alpha+1) \frac{\beta-1}{\beta} e^{-2 w(\alpha+1)}+c\left(\frac{1}{\beta}-2\right)(\alpha+1) e^{-w(\alpha+1)} w_{t}-w_{t t} \\
\left.-2 h \nabla^{2} w-2 \nabla w\left(\operatorname{div} h-\frac{1}{2} \nabla\left(\operatorname{tr}_{g} h\right)\right)+c(\alpha+1) e^{-w(\alpha+1)} \frac{F}{\beta t}\right),
\end{gathered}
$$


and by Lemma 2.1, we get

$$
\begin{aligned}
F_{t} & =\left(\beta|\nabla w|^{2}+c e^{-w(\alpha+1)}-w_{t}\right)+t\left(\beta\left(|\nabla w|^{2}\right)_{t}-c(\alpha+1) e^{-w(\alpha+1)} w_{t}-w_{t t}\right) \\
& =\frac{F}{t}+t\left(2 \beta \nabla w \nabla w_{t}-2 \beta h(\nabla w, \nabla w)-c(\alpha+1) e^{-w(\alpha+1)} w_{t}-w_{t t}\right) .
\end{aligned}
$$

Therefore, it follows that

$$
\begin{aligned}
& \left(\Delta-\frac{\partial}{\partial t}\right) F \\
& \geq-2 \nabla w \nabla F+t\left(2 \beta\left|\nabla^{2} w\right|^{2}+\left((2 \beta+\alpha-1) c(\alpha+1) e^{-w(\alpha+1)}\right)|\nabla w|^{2}\right. \\
& +c^{2}(\alpha+1) \frac{\beta-1}{\beta} e^{-2 w(\alpha+1)}-\frac{\beta-1}{\beta} c(\alpha+1) e^{-w(\alpha+1)} w_{t} \\
& -2 h \nabla^{2} w+2(\beta-1) K_{3}|\nabla w|^{2} \\
& \left.-2 K_{1} \beta|\nabla w|^{2}-2 \nabla w\left(\operatorname{div} h-\frac{1}{2} \nabla\left(\operatorname{tr}_{g} h\right)\right)\right) \\
& +c(\alpha+1) e^{-w(\alpha+1)} \frac{F}{\beta}-\frac{F}{t} \\
& =-2 \nabla w \nabla F+t\left(2 \beta\left|\nabla^{2} w\right|^{2}\right. \\
& +\left((\beta-1) c(\alpha+1) e^{-w(\alpha+1)}\right)\left(|\nabla w|^{2}+\frac{c e^{-w(\alpha+1)}}{\beta}-\frac{1}{\beta} w_{t}\right) \\
& +(\beta+\alpha) c(\alpha+1) e^{-w(\alpha+1)}|\nabla w|^{2}+2(\beta-1) K_{3}|\nabla w|^{2} \\
& \left.-2 K_{1} \beta|\nabla w|^{2}-2 h \nabla^{2} w-2 \nabla w\left(\operatorname{div} h-\frac{1}{2} \nabla\left(\operatorname{tr}_{g} h\right)\right)\right) \\
& +c(\alpha+1) e^{-w(\alpha+1)} \frac{F}{\beta}-\frac{F}{t} \\
& =-2 \nabla w \nabla F+t\left(2 \beta\left|\nabla^{2} w\right|^{2}+(\beta+\alpha) c(\alpha+1) e^{-w(\alpha+1)}|\nabla w|^{2}\right. \\
& +(\beta-1) c(\alpha+1) e^{-w(\alpha+1)} \frac{F}{\beta t}+2(\beta-1) K_{3}|\nabla w|^{2} \\
& \left.-2 K_{1} \beta|\nabla w|^{2}-2 h \nabla^{2} w-2 \nabla w\left(\operatorname{div} h-\frac{1}{2} \nabla\left(\operatorname{tr}_{g} h\right)\right)\right) \\
& +c(\alpha+1) e^{-w(\alpha+1)} \frac{F}{\beta}-\frac{F}{t} \\
& =-2 \nabla w \nabla F+t\left(2 \beta\left|\nabla^{2} w\right|^{2}+(\beta+\alpha) c(\alpha+1) e^{-w(\alpha+1)}|\nabla w|^{2}\right. \\
& +2(\beta-1) K_{3}|\nabla w|^{2}-2 K_{1} \beta|\nabla w|^{2} \\
& \left.-2 h \nabla^{2} w-2 \nabla w\left(\operatorname{div} h-\frac{1}{2} \nabla\left(\operatorname{tr}_{g} h\right)\right)\right) \\
& +c(\alpha+1) e^{-w(\alpha+1)} F-\frac{F}{t} .
\end{aligned}
$$


By our assumption, we have

$$
-\left(K_{2}+K_{3}\right) g \leq h \leq\left(K_{2}+K_{3}\right) g,
$$

which implies that

$$
|h|^{2} \leq\left(K_{2}+K_{3}\right)^{2}|g|^{2}=n\left(K_{2}+K_{3}\right)^{2} .
$$

Applying those bounds and Young's inequality yields

$$
\left|h \nabla^{2} w\right| \leq \frac{\beta}{2}\left|\nabla^{2} w\right|^{2}+\frac{1}{2 \beta}|h|^{2} \leq \frac{\beta}{2}\left|\nabla^{2} w\right|^{2}+\frac{n}{2 \beta}\left(K_{2}+K_{3}\right)^{2} .
$$

On the other hand,

$$
\left|\operatorname{div} h-\frac{1}{2} \nabla\left(\operatorname{tr}_{g} h\right)\right|=\left|g^{i j} \nabla_{i} h_{j l}-\frac{1}{2} g^{i j} \nabla_{l} h_{i j}\right| \leq \frac{3}{2}|g||\nabla h| \leq \frac{3}{2} \sqrt{n} K_{4} .
$$

Finally, with the help of the inequality

$$
\left|\nabla^{2} w\right|^{2} \geq \frac{1}{n}\left(\operatorname{tr} \nabla^{2} w\right)^{2}=\frac{1}{n}(\Delta w)^{2}=\frac{1}{n}\left(|\nabla w|^{2}+c(\alpha+1) e^{-w(\alpha+1)}-w_{t}\right)^{2},
$$

we get

$$
\begin{aligned}
& \left(\Delta-\frac{\partial}{\partial t}\right) F \\
& \geq-2 \nabla w \nabla F+t\left(\frac{\beta}{n}|\Delta w|^{2}+(\beta+\alpha) c(\alpha+1) e^{-w(\alpha+1)}|\nabla w|^{2}+2(\beta-1) K_{3}|\nabla w|^{2}\right. \\
& \left.\quad-2 K_{1} \beta|\nabla w|^{2}-\frac{n}{\beta}\left(K_{2}+K_{3}\right)^{2}-3 \sqrt{n} K_{4}|\nabla w|\right) \\
& \quad+c(\alpha+1) e^{-w(\alpha+1)} F-\frac{F}{t} .
\end{aligned}
$$

Since

$$
3 \sqrt{n} K_{4}|\nabla w| \leq 3 K_{4}\left(\frac{1}{2} n+\frac{1}{2}|\nabla w|^{2}\right),
$$

we have

$$
\begin{aligned}
& \left(\Delta-\frac{\partial}{\partial t}\right) F \\
& \geq-2 \nabla w \nabla F+t\left(\frac{\beta}{n}\left(|\nabla w|^{2}+c(\alpha+1) e^{-w(\alpha+1)}-w_{t}\right)^{2}\right. \\
& +\left((\beta+\alpha) c(\alpha+1) e^{-w(\alpha+1)}+2(\beta-1) K_{3}-2 \beta K_{1}-\frac{3}{2} K_{4}\right)|\nabla w|^{2} \\
& \left.-n\left(\frac{1}{\beta}\left(K_{2}+K_{3}\right)^{2}+\frac{3}{2} K_{4}\right)\right) \\
& +c(\alpha+1) e^{-w(\alpha+1)} F-\frac{F}{t} .
\end{aligned}
$$

This completes the proof of Lemma 2.2. 
Remark. If the general geometric flow is Ricci flow, that is, if $h=$ const $\cdot$ Ric $g$, the term $\operatorname{div} h-\frac{1}{2} \nabla\left(\operatorname{tr}_{g} h\right)$ in the above proof will vanish.

We take a $C^{2}$ cutoff function $\tilde{\varphi}$ defined on $[0, \infty)$ such that $\tilde{\varphi}(r)=1$ for $r \in[0,1]$, $\tilde{\varphi}(r)=0$ for $r \in[2, \infty)$, and $0 \leq \tilde{\varphi}(r) \leq 1$. Furthermore $\tilde{\varphi}$ satisfies

$$
-\frac{\tilde{\varphi}^{\prime}(r)}{\tilde{\varphi}^{1 / 2}(r)} \leq c_{1}
$$

and

$$
\tilde{\varphi}^{\prime \prime}(r) \geq-c_{2}
$$

for two constants $c_{1}, c_{2}>0$. Set

$$
\varphi(x, t)=\tilde{\varphi}\left(\frac{r(x, t)}{R}\right),
$$

where $r(x, t)=d\left(x, x_{0}, t\right)$. Using an argument of Calabi [1958], we can assume $\varphi(x, t) \in C^{2}(M)$ with support in $Q_{2 R, T}$. Direct calculation shows that on $Q_{2 R, T}$

$$
\frac{|\nabla \varphi|^{2}}{\varphi} \leq \frac{c_{1}^{2}}{R^{2}}
$$

By the Laplacian comparison theorem in [Aubin 1982],

$$
\Delta \varphi \geq-\frac{(n-1)\left(1+\sqrt{K_{1}} R\right) c_{1}^{2}+c_{2}}{R^{2}} .
$$

For any $0<T_{1}<T$, let $\left(x_{0}, t_{0}\right)$ be a point in the cube $Q_{2 R, T_{1}}$ at which $\varphi F$ attains its maximum value. We can assume that this value is positive (otherwise the proof is trivial). At the point $\left(x_{0}, t_{0}\right)$, we have

$$
\nabla(\varphi F)=0, \quad \Delta(\varphi F) \leq 0, \quad(\varphi F)_{t} \geq 0 .
$$

It follows that

$$
0 \geq\left(\Delta-\frac{\partial}{\partial t}\right)(\varphi F)=(\Delta \varphi) F-\varphi_{t} F+\varphi\left(\Delta-\frac{\partial}{\partial t}\right) F+2 \nabla \varphi \nabla F .
$$

By [Sun 2011, p. 494], we know there exists a positive constant $c_{3}$ such that

$$
-\varphi_{t} F \geq-\sqrt{c_{3}} K_{2} F \text {. }
$$

So we obtain

$$
\varphi\left(\Delta-\frac{\partial}{\partial t}\right) F+F \Delta \varphi-\varphi_{t} F-2 F \varphi^{-1}|\nabla \varphi|^{2} \leq 0 .
$$

This inequality, together with the inequalities (2-2) and (2-3), yields

$$
\varphi\left(\Delta-\frac{\partial}{\partial t}\right) F \leq A F,
$$


where

$$
A=\frac{\left((n-1)\left(1+\sqrt{K_{1}} R\right)\right) c_{1}^{2}+c_{2}+2 c_{1}^{2}}{R^{2}}+\sqrt{c_{3}} K_{2} .
$$

At $\left(x_{0}, t_{0}\right)$, by Lemma 2.2, we have

$$
\begin{aligned}
& 0 \geq \varphi\left(\Delta-\frac{\partial}{\partial t}\right) F-A F \\
& \geq-A F+\varphi\left(-\frac{F}{t_{0}}+c(\alpha+1) e^{-w(\alpha+1)} F+\frac{\beta t_{0}}{n}\left(|\nabla w|^{2}+c(\alpha+1) e^{-w(\alpha+1)}-w_{t}\right)^{2}\right. \\
& \quad-2 \nabla w \nabla F \\
& \quad+\left((\beta+\alpha) c(\alpha+1) e^{-w(\alpha+1)}+2(\beta-1) K_{3}-2 \beta K_{1}-\frac{3}{2} K_{4}\right)|\nabla w|^{2} t_{0} \\
&\left.\quad-n\left(\frac{1}{\beta}\left(K_{2}+K_{3}\right)^{2}+\frac{3}{2} K_{4}\right) t_{0}\right) .
\end{aligned}
$$

(1) If $c<0$ and $u^{-(\alpha+1)} \leq \tilde{M}$ for all $(x, t) \in M \times[0, T]$, we have

$$
\begin{aligned}
0 \geq & -A F-\varphi \frac{F}{t_{0}}+\varphi \frac{\beta t_{0}}{n}\left(|\nabla w|^{2}+c(\alpha+1) e^{-w(\alpha+1)}-w_{t}\right)^{2}-2 \varphi \nabla w \nabla F \\
& +c \tilde{M}(\alpha+1) \varphi F+\left((\beta+\alpha) c(\alpha+1) \tilde{M}+2(\beta-1) K_{3}-2 \beta K_{1}-\frac{3}{2} K_{4}\right)|\nabla w|^{2} \varphi t_{0} \\
& -\left(\frac{1}{\beta}\left(K_{2}+K_{3}\right)^{2}+\frac{3}{2} K_{4}\right) n \varphi t_{0} .
\end{aligned}
$$

Set

$$
\widetilde{C}_{1}=-(\beta+\alpha) c(\alpha+1) \tilde{M}+2(1-\beta) K_{3}+2 \beta K_{1}+\frac{3}{2} K_{4}
$$

and

$$
\widetilde{C}_{2}=\frac{1}{\beta}\left(K_{2}+K_{3}\right)^{2}+\frac{3}{2} K_{4} .
$$

Multiplying by $\varphi t_{0}$ on both sides of the above inequality, we get

$$
\begin{aligned}
0 \geq- & A \varphi t_{0} F-\varphi F+2 t_{0} F \varphi \nabla w \nabla \varphi+c \tilde{M}(\alpha+1) \varphi F t_{0} \\
& \quad-\widetilde{C}_{1}|\nabla w|^{2} \varphi^{2} t_{0}^{2}-\widetilde{C}_{2} n \varphi^{2} t_{0}^{2}+\varphi^{2} t_{0}^{2} \frac{\beta t_{0}}{n}\left(|\nabla w|^{2}+c(\alpha+1) e^{-w(\alpha+1)}-w_{t}\right)^{2} \\
\geq \varphi & F\left(-A t_{0}-1+c \tilde{M}(\alpha+1) t_{0}\right)-\frac{2 c_{1}}{R} t_{0} F \varphi^{3 / 2}|\nabla w| \\
& \quad+\frac{\beta t_{0}^{2}}{n}\left(\varphi^{2}\left(|\nabla w|^{2}+c(\alpha+1) e^{-w(\alpha+1)}-w_{t}\right)^{2}-\frac{n}{\beta} \widetilde{C}_{1} \varphi^{2}|\nabla w|^{2}\right)-\widetilde{C}_{2} n t_{0}^{2},
\end{aligned}
$$

where the last inequality used

$$
-2 \varphi \nabla w \nabla F=2 F \nabla w \nabla \varphi \geq-2 F|\nabla w||\nabla \varphi| \geq-\frac{2 c_{1}}{R} \varphi^{1 / 2} F|\nabla w| .
$$


Assume that $y=\varphi|\nabla w|^{2}$ and $z=\varphi\left(-c e^{-w(\alpha+1)}+w_{t}\right)$. We have

$0 \geq \varphi F\left(-A t_{0}-1+c \tilde{M}(\alpha+1) t_{0}\right)$

$$
+\frac{\beta t_{0}^{2}}{n}\left((y-z)^{2}-\frac{n}{\beta} \widetilde{C}_{1} y-2 \frac{n c_{1}}{R} y^{1 / 2}\left(y-\frac{z}{\beta}\right)\right)-\widetilde{C}_{2} n t_{0}^{2} .
$$

Using the inequality $a x^{2}-b x \geq-b^{2} /(4 a)$, valid for $a, b>0$, one obtains

$$
\begin{aligned}
& \frac{\beta t_{0}^{2}}{n}\left((y-z)^{2}-\frac{n}{\beta} \widetilde{C}_{1} y-2 \frac{n c_{1}}{R} y^{1 / 2}\left(y-\frac{z}{\beta}\right)\right) \\
& =\frac{\beta t_{0}^{2}}{n}\left(\beta^{2}\left(y-\frac{z}{\beta}\right)^{2}+(1-\beta)^{2} y^{2}-\frac{n}{\beta} \widetilde{C}_{1} y+\left(2\left(\beta-\beta^{2}\right) y-2 \frac{n c_{1}}{R} y^{1 / 2}\right)\left(y-\frac{z}{\beta}\right)\right) \\
& \geq \frac{\beta t_{0}^{2}}{n}\left(\beta^{2}\left(y-\frac{z}{\beta}\right)^{2}-\frac{n^{2} \widetilde{C}_{1}^{2}}{4 \beta^{2}(1-\beta)^{2}}-\frac{n^{2} c_{1}^{2}}{2 R^{2}\left(\beta-\beta^{2}\right)}\left(y-\frac{z}{\beta}\right)\right) \\
& =\frac{\beta}{n}(\varphi F)^{2}-\frac{n \widetilde{C}_{1}^{2} t_{0}^{2}}{4 \beta(1-\beta)^{2}}-\frac{n c_{1}^{2} t_{0}}{2 R^{2}\left(\beta-\beta^{2}\right)}(\varphi F) .
\end{aligned}
$$

Hence,

$$
\begin{aligned}
\frac{\beta}{n}(\varphi F)^{2}+\left(-A t_{0}-1+c \tilde{M}(\alpha+1) t_{0}-\frac{n c_{1}^{2} t_{0}}{2 R^{2}\left(\beta-\beta^{2}\right)}\right) & (\varphi F) \\
& -\frac{n \widetilde{C}_{1}^{2} t_{0}^{2}}{4 \beta(1-\beta)^{2}}-\widetilde{C}_{2} n t_{0}^{2} \leq 0 .
\end{aligned}
$$

From the inequality $A x^{2}-2 B x \leq C$, we have $x \leq 2 B / A+\sqrt{C / A}$. We can get $\varphi F \leq \frac{n}{\beta}\left(A t_{0}+1-c \tilde{M}(\alpha+1) t_{0}+\frac{n c_{1}^{2} t_{0}}{2 R^{2}\left(\beta-\beta^{2}\right)}\right)+\left(\frac{n}{\beta}\left(\frac{n \widetilde{C}_{1}^{2}}{4 \beta(1-\beta)^{2}}+\widetilde{C}_{2} n\right)\right)^{\frac{1}{2}} t_{0}$ If $d\left(x, x_{0}, T_{1}\right)<R$, we have $\varphi\left(x, T_{1}\right)=1$. Then

$$
\begin{aligned}
F & \left(x, T_{1}\right) \\
& =T_{1}\left(\beta|\nabla w|^{2}+c e^{-w(\alpha+1)}-w_{t}\right) \\
& \leq \varphi F\left(x_{0}, t_{0}\right) \\
& \leq \frac{n}{\beta}\left(A t_{0}+1-c \tilde{M}(\alpha+1) t_{0}+\frac{n c_{1}^{2} t_{0}}{2 R^{2}\left(\beta-\beta^{2}\right)}\right)+\left(\frac{n}{\beta}\left(\frac{n \widetilde{C}_{1}^{2}}{4 \beta(1-\beta)^{2}}+\widetilde{C}_{2} n\right)\right)^{\frac{1}{2}} t_{0} .
\end{aligned}
$$

As $T_{1}$ is arbitrary, we can get case (1) of Theorem 1.3. 
(2) If $c>0$, we have

$$
\begin{aligned}
0 \geq-A F+\varphi & \left(-\frac{F}{t_{0}}+\frac{\beta t_{0}}{n}\left(|\nabla w|^{2}+c(\alpha+1) e^{-w(\alpha+1)}-w_{t}\right)^{2}-2 \nabla w \nabla F\right. \\
& \left.+\left(2(\beta-1) K_{3}-2 \beta K_{1}-\frac{3}{2} K_{4}\right)|\nabla w|^{2} t_{0}-n\left(\frac{1}{\beta}\left(K_{2}+K_{3}\right)^{2}+\frac{3}{2} K_{4}\right) t_{0}\right) .
\end{aligned}
$$

Therefore,

$$
\begin{array}{rl}
0 \geq-A & F-\varphi \frac{F}{t_{0}}+\varphi \frac{\beta t_{0}}{n}\left(|\nabla w|^{2}+c(\alpha+1) e^{-w(\alpha+1)}-w_{t}\right)^{2}-2 \varphi \nabla w \nabla F \\
& +\left(2(\beta-1) K_{3}-2 \beta K_{1}-\frac{3}{2} K_{4}\right)|\nabla w|^{2} \varphi t_{0}-\left(\frac{1}{\beta}\left(K_{2}+K_{3}\right)^{2}+\frac{3}{2} K_{4}\right) n \varphi t_{0} .
\end{array}
$$

Similarly, we can get case (2) of Theorem 1.3. This completes the proof of Theorem 1.3.

Proof of Theorem 1.5. For any points $\left(x_{1}, t_{1}\right)$ and $\left(x_{2}, t_{2}\right)$ on $M \times(0, T]$ with $0<t_{1}<t_{2}$, we take a curve $\gamma(t)$ parametrized with $\gamma\left(t_{1}\right)=x_{1}$ and $\gamma\left(t_{2}\right)=x_{2}$. One gets from Corollary 1.4:

(1) If $c<0$, we have

$$
\begin{aligned}
\log u\left(x_{2}, t_{2}\right)- & \log u\left(x_{1}, t_{1}\right) \\
& =\int_{t_{1}}^{t_{2}}\left((\log u)_{t}+\langle\nabla \log u, \dot{\gamma}\rangle\right) d t \\
& \geq \int_{t_{1}}^{t_{2}}\left(\beta|\nabla \log u|^{2}-\frac{n}{\beta t}-c u^{-(\alpha+1)}-\bar{H}_{1}-H_{2}-|\nabla \log u||\dot{\gamma}|\right) d t \\
& \geq-\int_{t_{1}}^{t_{2}}\left(\frac{1}{4 \beta}|\dot{\gamma}|^{2}+\frac{n}{\beta t}+\bar{H}_{1}+H_{2}\right) d t \\
& =-\left(\int_{t_{1}}^{t_{2}} \frac{1}{4 \beta}|\dot{\gamma}|^{2} d t+\log \left(\frac{t_{2}}{t_{1}}\right)^{n / \beta}+\left(\bar{H}_{1}+H_{2}\right)\left(t_{2}-t_{1}\right)\right),
\end{aligned}
$$

which means that

$$
\log \frac{u\left(x_{1}, t_{1}\right)}{u\left(x_{2}, t_{2}\right)} \leq \int_{t_{1}}^{t_{2}} \frac{1}{4 \beta}|\dot{\gamma}|^{2} d t+\log \left(\frac{t_{2}}{t_{1}}\right)^{n / \beta}+\left(\bar{H}_{1}+H_{2}\right)\left(t_{2}-t_{1}\right) .
$$

Therefore,

$$
u\left(x_{1}, t_{1}\right) \leq u\left(x_{2}, t_{2}\right)\left(\frac{t_{2}}{t_{1}}\right)^{n / \beta} e^{\varphi\left(x_{1}, x_{2}, t_{1}, t_{2}\right)+\left(\bar{H}_{1}+H_{2}\right)\left(t_{2}-t_{1}\right)},
$$

where $\varphi\left(x_{1}, x_{2}, t_{1}, t_{2}\right)=\inf _{\gamma} \int_{t_{1}}^{t_{2}} \frac{1}{4 \beta}|\dot{\gamma}|^{2} d t$. 
(2) If $c>0$, we have

$\log u\left(x_{2}, t_{2}\right)-\log u\left(x_{1}, t_{1}\right)$

$$
\begin{aligned}
& =\int_{t_{1}}^{t_{2}}\left((\log u)_{t}+\langle\nabla \log u, \dot{\gamma}\rangle\right) d t \\
& \geq \int_{t_{1}}^{t_{2}}\left(\beta|\nabla \log u|^{2}-\frac{n}{\beta t}-c u^{-(\alpha+1)}-\widehat{H}_{1}-\widetilde{H}_{2}-|\nabla \log u||\dot{\gamma}|\right) d t \\
& \geq-\int_{t_{1}}^{t_{2}}\left(\frac{1}{4 \beta}|\dot{\gamma}|^{2}+\frac{n}{\beta t}+c \tilde{M}+\hat{H}_{1}+\tilde{H}_{2}\right) d t \\
& =-\left(\int_{t_{1}}^{t_{2}} \frac{1}{4 \beta}|\dot{\gamma}|^{2} d t+\log \left(\frac{t_{2}}{t_{1}}\right)^{n / \beta}+\left(c \tilde{M}+\hat{H}_{1}+\tilde{H}_{2}\right)\left(t_{2}-t_{1}\right)\right),
\end{aligned}
$$

which means that

$$
\log \frac{u\left(x_{1}, t_{1}\right)}{u\left(x_{2}, t_{2}\right)} \leq \int_{t_{1}}^{t_{2}} \frac{1}{4 \beta}|\dot{\gamma}|^{2} d t+\log \left(\frac{t_{2}}{t_{1}}\right)^{n / \beta}+\left(c \tilde{M}+\hat{H}_{1}+\tilde{H}_{2}\right)\left(t_{2}-t_{1}\right) .
$$

Therefore,

$$
u\left(x_{1}, t_{1}\right) \leq u\left(x_{2}, t_{2}\right)\left(\frac{t_{2}}{t_{1}}\right)^{n / \beta} e^{\varphi\left(x_{1}, x_{2}, t_{1}, t_{2}\right)+\left(c \tilde{M}+\hat{H}_{1}+\tilde{H}_{2}\right)\left(t_{2}-t_{1}\right)},
$$

where $\varphi\left(x_{1}, x_{2}, t_{1}, t_{2}\right)=\inf _{\gamma} \int_{t_{1}}^{t_{2}} \frac{1}{4 \beta}|\dot{\gamma}|^{2} d t$.

\section{Acknowledgements}

The authors would like to thank their supervisor, Professor Kefeng Liu, for his constant encouragement and help. Zhao was supported in part by the Natural Science Foundation of Jiangsu Province (BK20140804) and the Fundamental Research Funds for the Central Universities (NS2014076). Fang was supported by NSFC (11401514 and 11371310) and the Natural Science Foundation of the Jiangsu Higher Education Institutions of China (13KJB110029).

\section{References}

[Aubin 1982] T. Aubin, Nonlinear analysis on manifolds. Monge-Ampère equations, Grundlehren der Mathematischen Wissenschaften 252, Springer, New York, 1982. MR 681859 Zbl 0512.53044

[Calabi 1958] E. Calabi, "An extension of E. Hopf's maximum principle with an application to Riemannian geometry”, Duke Math. J. 25 (1958), 45-56. MR 0092069 Zbl 0079.11801

[Chen and Chen 2009] L. Chen and W. Chen, "Gradient estimates for a nonlinear parabolic equation on complete non-compact Riemannian manifolds", Ann. Global Anal. Geom. 35:4 (2009), 397-404. MR 2506242 Zbl 1177.35040

[Chen and Chen 2010] L. Chen and W. Chen, "Gradient estimates for positive smooth $f$-harmonic functions”, Acta Math. Sci. Ser. B Engl. Ed. 30:5 (2010), 1614-1618. MR 2778630 Zbl 1240.58019 
[Huang and Li 2014] G. Huang and H. Li, "Gradient estimates and entropy formulae of porous medium and fast diffusion equations for the Witten Laplacian”, Pacific J. Math. 268:1 (2014), 47-78. MR 3207600 Zbl 1297.35059

[Kuang and Zhang 2008] S. Kuang and Q. S. Zhang, "A gradient estimate for all positive solutions of the conjugate heat equation under Ricci flow", J. Funct. Anal. 255:4 (2008), 1008-1023. MR 2433960 Zbl 1146.58017

[Li 2005] X.-D. Li, "Liouville theorems for symmetric diffusion operators on complete Riemannian manifolds", J. Math. Pures Appl. (9) 84:10 (2005), 1295-1361. MR 2170766 Zbl 1082.58036

[Liu 2009] S. Liu, "Gradient estimates for solutions of the heat equation under Ricci flow", Pacific J. Math. 243:1 (2009), 165-180. MR 2550141 Zbl 1180.58017

[Ma 2006] L. Ma, "Gradient estimates for a simple elliptic equation on complete non-compact Riemannian manifolds", J. Funct. Anal. 241:1 (2006), 374-382. MR 2264255 Zbl 1112.58023

[Ma 2010] L. Ma, "Hamilton type estimates for heat equations on manifolds", preprint, 2010. arXiv 1009.0603

[Perelman 2002] G. Perelman, "The entropy formula for the Ricci flow and its geometric applications", preprint, 2002. arXiv math/0211159

[Sun 2011] J. Sun, "Gradient estimates for positive solutions of the heat equation under geometric flow”, Pacific J. Math. 253:2 (2011), 489-510. MR 2878821 Zbl 1235.53070

[Wu 2010] J.-Y. Wu, "Li-Yau type estimates for a nonlinear parabolic equation on complete manifolds", J. Math. Anal. Appl. 369:1 (2010), 400-407. MR 2643878 Zbl 1211.58017

[Zhang and Ma 2011] J. Zhang and B. Ma, "Gradient estimates for a nonlinear equation $\Delta_{f} u+$ $c u^{-\alpha}=0$ on complete noncompact manifolds", Commun. Math. 19:1 (2011), 73-84. MR 2855392 Zbl 1242.58011

[Zhao 2013] L. Zhao, "Harnack inequality for parabolic Lichnerowicz equations on complete noncompact Riemannian manifolds”, Bound. Value Probl. 2013 (2013), 190, 10. MR 3117294 Zbl 1298.58016

[Zhao 2014] L. Zhao, "Gradient estimates for a simple parabolic Lichnerowicz equation", Osaka J. Math. 51:1 (2014), 245-256. MR 3192542 Zbl 1296.58016

[Zhu 2011] X. Zhu, "Hamilton's gradient estimates and Liouville theorems for fast diffusion equations on noncompact Riemannian manifolds", Proc. Amer. Math. Soc. 139:5 (2011), 1637-1644. MR 2763753 Zbl 1217.35041

Received September 16, 2015. Revised April 20, 2016.

\section{LIANG ZHAO}

DEPARTMENT OF MATHEMATICS

NANJing UniVERSity OF AERONAUTICS AND AstronaUtics

29 Yudao StreEt, QINHUAi District

NANJING 210016

CHINA

zhaozongliang09@163.com

\section{SHOUWEN FANG}

SCHOOL of Mathematical SCIENCE

YANGZHOU UNIVERSITY

YANGZHOU 225002

CHINA

shwfang@163.com 


\title{
PACIFIC JOURNAL OF MATHEMATICS
}

Founded in 1951 by E. F. Beckenbach (1906-1982) and F. Wolf (1904-1989)

$$
\text { msp.org/pjm }
$$

\section{EDITORS}

\author{
Don Blasius (Managing Editor) \\ Department of Mathematics \\ University of California \\ Los Angeles, CA 90095-1555 \\ blasius@math.ucla.edu
}

\author{
Paul Balmer \\ Department of Mathematics \\ University of California \\ Los Angeles, CA 90095-1555 \\ balmer@math.ucla.edu \\ Robert Finn \\ Department of Mathematics \\ Stanford University \\ Stanford, CA 94305-2125 \\ finn@math.stanford.edu \\ Sorin Popa \\ Department of Mathematics \\ University of California \\ Los Angeles, CA 90095-1555 \\ popa@math.ucla.edu
}

\author{
Vyjayanthi Chari \\ Department of Mathematics \\ University of California \\ Riverside, CA 92521-0135 \\ chari@math.ucr.edu \\ Kefeng Liu \\ Department of Mathematics \\ University of California \\ Los Angeles, CA 90095-1555 \\ liu@math.ucla.edu \\ Igor Pak \\ Department of Mathematics \\ University of California \\ Los Angeles, CA 90095-1555 \\ pak.pjm@gmail.com \\ Paul Yang \\ Department of Mathematics \\ Princeton University \\ Princeton NJ 08544-1000 \\ yang@math.princeton.edu
}

\section{PRODUCTION}

Silvio Levy, Scientific Editor, production@msp.org

\section{SUPPORTING INSTITUTIONS}

ACADEMIA SINICA, TAIPEI

CALIFORNIA INST. OF TECHNOLOGY

STANFORD UNIVERSITY

UNIV. OF BRITISH COLUMBIA

UNIV. OF CALIFORNIA, BERKELEY

UNIV. OF CALIFORNIA, DAVIS

UNIV. OF CALIFORNIA, LOS ANGELES

UNIV. OF CALIFORNIA, RIVERSIDE

UNIV. OF CALIFORNIA, SAN DIEGO

UNIV. OF CALIF., SANTA BARBARA
KEIO UNIVERSITY

MATH. SCIENCES RESEARCH INSTITUTE

NEW MEXICO STATE UNIV.

OREGON STATE UNIV.
Daryl Cooper

Department of Mathematics

University of California

Santa Barbara, CA 93106-3080 cooper@math.ucsb.edu

Jiang-Hua Lu

Department of Mathematics

The University of Hong Kong

Pokfulam Rd., Hong Kong

jhlu@maths.hku.hk

$$
\text { Jie Qing }
$$

Department of Mathematics

University of California

Santa Cruz, CA 95064

qing@ cats.ucsc.edu

\author{
UNIV. OF CALIF., SANTA CRUZ \\ UNIV. OF MONTANA \\ UNIV. OF OREGON \\ UNIV. OF SOUTHERN CALIFORNIA \\ UNIV. OF UTAH \\ UNIV. OF WASHINGTON \\ WASHINGTON STATE UNIVERSITY
}

These supporting institutions contribute to the cost of publication of this Journal, but they are not owners or publishers and have no responsibility for its contents or policies.

See inside back cover or msp.org/pjm for submission instructions.

The subscription price for 2016 is US $\$ 440 /$ year for the electronic version, and \$600/year for print and electronic.

Subscriptions, requests for back issues and changes of subscriber address should be sent to Pacific Journal of Mathematics, P.O. Box 4163, Berkeley, CA 94704-0163, U.S.A. The Pacific Journal of Mathematics is indexed by Mathematical Reviews, Zentralblatt MATH, PASCAL CNRS Index, Referativnyi Zhurnal, Current Mathematical Publications and Web of Knowledge (Science Citation Index).

The Pacific Journal of Mathematics (ISSN 0030-8730) at the University of California, c/o Department of Mathematics, 798 Evans Hall \#3840, Berkeley, CA 94720-3840, is published twelve times a year. Periodical rate postage paid at Berkeley, CA 94704, and additional mailing offices. POSTMASTER: send address changes to Pacific Journal of Mathematics, P.O. Box 4163, Berkeley, CA 94704-0163.

PJM peer review and production are managed by EditFLOW ${ }^{\circledR}$ from Mathematical Sciences Publishers.

PUBLISHED BY

\section{I. mathematical sciences publishers}

nonprofit scientific publishing

http://msp.org/

(C) 2016 Mathematical Sciences Publishers 


\section{PACIFIC JOURNAL OF MATHEMATICS}

Volume $285 \quad$ No. $1 \quad$ November 2016

Iwahori-Hecke algebras for Kac-Moody groups over local fields

Nicole BARDY-PANSE, StÉPHANE GAUSSENT and GuY

ROUSSEAU

A classification of spherical conjugacy classes

MAURo Costantini

Affine weakly regular tensor triangulated categories

IVo DELL'AMBROGIO and DONALD STANLEY

Involutive automorphisms of $N_{\circ}^{\circ}$-groups of finite Morley rank

ADRIEN DELORO and ÉRIC JALIGOT

Schur-Weyl duality for Deligne categories, II: The limit case

INNA ENTOVA AIZENBUD

A generalization of the Greene-Krantz theorem for the semicontinuity 225 property of automorphism groups

JAE-CHEON JOO

Gradient estimates for a nonlinear Lichnerowicz equation under general geometric flow on complete noncompact manifolds

LIANG ZHAO and SHOUWEN FANG 